\title{
Caprin-1 promotes HepG2 cell proliferation, invasion and migration and is associated with poor prognosis in patients with liver cancer
}

\author{
XIN-MIN GUO ${ }^{1}$, FANG-FANG ZHU ${ }^{1}$, LI-WEN PAN ${ }^{2}$, JIA-LIN CHEN ${ }^{1}$, \\ JI-CHUANG LAI ${ }^{1}$, HONG-XIA WU ${ }^{1}$ and JIAN-CHANG SHU ${ }^{3}$ \\ Departments of ${ }^{1}$ Ultrasound, ${ }^{2}$ Endocrinology and ${ }^{3}$ Gastroenterology, Guangzhou Red Cross Hospital, \\ Medical College, Jinan University, Guangzhou, Guangdong 510220, P.R. China \\ Received May 7, 2019; Accepted February 14, 2020
}

DOI: $10.3892 / \mathrm{ol} .2020 .11712$

\begin{abstract}
The present study aimed to investigate the role of caprin-1 in liver cancer and its association with the clinicopathological features and prognosis of liver cancer, as well as the underlying mechanism of caprin-1 function. Caprin-1 expression levels in a tissue microarray containing 40 liver cancer tissues, 10 peritumoral tissues and 20 normal liver tissues were analyzed using immunohistochemistry. The clinical data of 154 patients with liver cancer were also collected from The Cancer Genome Atlas database. Kaplan-Meier analysis and a Cox proportional hazards regression model were used to assess the association between caprin-1 expression levels and survival in patients with liver cancer. The effects of caprin-1 knockdown on the mRNA levels of cyclin D1 and cyclin D2 as well as the proliferation, invasion and migration of HepG2 cells were also investigated. The expression level of caprin-1 in liver cancer tissues was significantly higher compared with normal liver tissues or cells $(\mathrm{P}<0.01)$. High caprin-1 expression levels were associated with advanced clinical stage $(\mathrm{P}<0.001)$ and enhanced tumor invasion $(\mathrm{P}<0.001)$. Kaplan-Meier analysis showed that the overall survival time and disease-free survival time in patients with liver cancer with high caprin-1 expression were significantly shorter compared with patients with low caprin-1 expression levels $(\mathrm{P}=0.002$ and $\mathrm{P}=0.033$, respectively). The Cox proportional hazards regression model
\end{abstract}

Correspondence to: Dr Xin-Min Guo, Department of Ultrasound, Guangzhou Red Cross Hospital, Medical College, Jinan University, 396 Tongfu Middle Road, Guangzhou, Guangdong 510220, P.R. China

E-mail: guoxm1509257@163.com

Dr Jian-Chang Shu, Department of Gastroenterology, Guangzhou Red Cross Hospital, Medical College, Jinan University, 396 Tongfu Middle Road, Guangzhou, Guangdong 510220, P.R. China E-mail: shujc0328@163.com

Key words: caprin-1, prognosis, cell proliferation, invasion and migration showed that high caprin-1 expression levels were an independent prognostic factor for liver cancer $(\mathrm{P}<0.001)$. Knockdown of caprin-1 in HepG2 cells significantly downregulated mRNA expression levels of cyclin D1 and cyclin D2, inhibited cell proliferation and invasion and the cells were arrested at $\mathrm{G}_{0} / \mathrm{G}_{1}$ phase. In conclusion, caprin-1 may be a novel prognostic indicator for patients with liver cancer.

\section{Introduction}

Liver cancer is currently the sixth most common cancer and the third leading cause of cancer-associated death worldwide in $2013(1,2)$. The number of liver cancer-associated deaths increased from $\sim 510,100$ in 1993 to 818,000 in 2013 globally $(3,4)$. At present, surgery, liver transplantation, radiofrequency ablation, interventional therapy, gene therapy and immunotherapy are available for treatment of liver cancer (5). Of these options, surgical treatment, including partial liver tissue resection and liver transplantation, is currently the best treatment option for liver cancer with a 5-year overall survival rate of $>70 \%$ for early-stage liver cancer (6-8) and 25-39\% for liver cancer of all stages worldwide (9). However, surgical resection is feasible in only $10-37 \%$ of liver cancer cases as patients with liver cancer are typically diagnosed at an advanced stage worldwide (10). Moreover, the long-term overall survival rate following surgery remains unsatisfactory due to the high recurrence and metastasis rates $(50-60 \%)(9,11)$. Therefore, diagnosis of liver cancer during an early stage, and the ability to predict the prognosis, recurrence and metastasis of liver cancer following treatment is important for improving the survival of patients.

In contrast to traditional clinicopathological indicators, biomarkers are more advantageous for identifying patients with a higher risk of recurrence or metastasis (12). Although several biomarkers, such as glypican-3 (13), CpG island methylator phenotype (14), neutrophil-lymphocyte ratio (10), platelet-lymphocyte ratio (15) have been reported to be prognostic markers for liver cancer recurrence or progression after treatment, their clinical application remains unsatisfactory because of their limited prognostic value, especially in patients with advanced stage disease (10,12-15). Therefore, identifying 
novel prognostic biomarkers for liver cancer is important and maybe beneficial in adjuvant therapy and improving patient survival.

Caprin-1 is a ubiquitously expressed, highly conserved cytoplasmic phosphoprotein in vertebrates and is associated with cell proliferation in all tissues except the brain (16). Caprin-1 expression is decreased when cells stop proliferating and begin differentiation and is increased when cells proliferate again (17). A lack of caprin-1 in cells can lead to a delay in the progression from $\mathrm{G}_{1}$ phase to the $S$ phase of the cell cycle (17). The carboxy-terminal arginine/glycine (RGG)-rich region of caprin-1 can selectively bind the mRNA encoding c-Myc and cyclin D2, which are involved in the regulation of cell proliferation (18) and can also directly bind to RasGTPase-activating protein-binding protein 1 to promote mammalian stress particle formation (19). In addition, overexpression of caprin-1 can lead to overall inhibition of stress granule formation in cells (19). Caprin-1 and helicase colocalize in the leading edge of migrating fibroblasts to promote cell migration and spreading (20). Several studies have reported that caprin-1 overexpression is associated with the occurrence and development of tumors, such as osteosarcoma, breast cancer and colon cancer (21-23).

The present study aimed to investigate the role of caprin-1 in liver cancer and its association with the clinicopathological features and prognosis in patients with liver cancer, as well as the underlying mechanism of caprin-1 function in liver cancer.

\section{Materials and methods}

Patients and tissue samples. A tissue microarray (TMA; $\mathrm{n}=70$ ), including 40 liver cancer samples (One liver cancer sample was excluded as the IHC results revealed it was an interstitial tissue), 10 specimens of paired adjacent normal liver tissue, the distance of which to the tumor tissue was $\sim 4 \mathrm{~cm}$, and 20 specimens of normal liver tissue, together with detailed clinical information were purchased from Xi'an Alenabio Co., Ltd. (cat. no. BC03116a;) and used for immunohistochemical analysis. The liver histology and morphology of patients was observed using hematoxylin and eosin staining performed by Pathology department of Guangzhou Red Cross Hospital (Guangzhou, China). The inclusion criteria were patients pathologically diagnosed with liver cancer. The exclusion criteria included patients who had received chemotherapy or radiation therapy before surgery. The clinical information of 134 patients with liver cancer was also collected from The Cancer Genome Atlas (TCGA) database downloaded from Cbioprotal (cbioportal.org/) to investigate the mRNA expression levels of caprin-1 and to perform survival analysis. The clinical stages of these patients were divided according to American Joint Committee on Cancer staging manual (24).

Immunohistochemistry (IHC). Specimens were fixed in $10 \%$ formalin at room temperature for $48 \mathrm{~h}$ and subsequently embedded in paraffin. The paraffin-embedded tissues were cut into $4-\mu \mathrm{m}$ sections, then deparaffinized with xylene and rehydrated using aDAKO EnVisionsystem (Dako; Agilent Technologies, Inc.). IHC staining was carried out using the UltraSensitive ${ }^{\mathrm{TM}} \mathrm{SP}$ (mouse/rabbit) IHC kit (catalog no. KIT-0305; MX Biotechnologies) which included endogenous peroxidase blocking solution, serum, secondary antibody, streptavidin-peroxidase and DAB substrate-chromogen. The tissue slice was added with $50 \mu \mathrm{l}$ peroxidase blocking solution to block the activity of endogenous peroxidase, and incubated at room temperature for 15 min. Rinsed with PBS 3 times with 3 min each time. Blocking was performed with goat serum UltraSensitive ${ }^{\mathrm{TM}}$ SP (mouse/rabbit) IHC kit for $30 \mathrm{~min}$ at room temperature. Then the slides were incubated with Caprin-1 rabbit polyclonal antibody (1:100 dilution; ab38859; Abcam) overnight at $4^{\circ} \mathrm{C}$. After washing with PBS, the slides were incubated with $50 \mu \mathrm{l}$ streptomyces antibiotin-peroxidase solution for $15 \mathrm{~min}$ at room temperature and then soaked in the $100 \mu l$ diaminobenzidine for $2 \mathrm{~min}$ at room temperature. One slide not incubated with caprin-1 antibody was used as a negative control. The intensity of immunostaining was scored independently by two experienced pathologists, who were blinded to the patient's clinical pathology data and clinical outcomes. The scores were determined and compared by the same pathologist. Under the light microscope, the numbers of positively stained cells in five representative regions at $\times 400$ magnification were counted and the mean percentage of positive cells was calculated. Cytoplasmic staining was considered as a positive signal according to the manufacturer's protocol. The staining intensity was stratified and scored according to the following criteria: No staining, 0 points; mild staining, 1 point; medium staining, 2 points; and strong staining, 3 points. Scores for the percentage of immunostaining tumor cells were defined as follows: $<5 \%, 0$ Points; $6-25 \%, 1$ point; $26-50 \%, 2$ points; $51-75 \%, 3$ points; and $>75 \%, 4$ points. The final immune response score (IRS) for each case was calculated by multiplying the two scores for the immunostaining intensity and the percentage of immunostaining. The expression of caprin-1 was divided into two groups: Low expression level $(<$ mean expression levels of caprin-1), and high expression level (>mean expression levels of caprin-1).

Cells and plasmid. Human HepG2 liver cancer cell line (Guangzhou Hunyuanyuan Medical Technology Co., Ltd.) was cultured in Dulbecco's Modified Eagle's Medium (DMEM; Hyclone; GE Healthcare Life Science) with $10 \%$ fetal bovine serum (FBS; Hyclone; GE Healthcare Life Science). The caprin-1 knockout (KO) plasmids PXC9-puro-KO-1 and PXC9-puro-KO-2 was constructed by inserting cDNA of siRNA targeting caprin-1 5'-CACCGCGACAAGAAACTT CGGAACCGTTT-3' (KO-1) or 5'-CACCGGTCCGGACCG CCACCGCCGTGTTT-3' (KO-2) into the BsmBI site of PXC9-puro (cat. no. 52961; Addgene, Inc.). The recombinant plasmids were confirmed by sequencing (Synbio Technologies LLC). The knockdown effect of the plasmids was detected using western blot analysis and PXC9-puro-KO-2 was selected for further experiments. The plasmid PXC9-puro-NC sequence was 5'-GTAGGCGCGCCGCTCTCTAC-3' and was a non-targeting control guide used as a negative control.

Transfection using ultrasound treatment. HepG2 cells were seeded in 24-well plates at $1 \times 10^{4}$ cells/well and cultured in medium containing $10 \%$ FBS until the cell confluence reached $60-70 \%$. Transfection of the recombinant plasmids was performed using ultrasonic irradiation treatment. Cells were 
incubated with a mixture of 5\% sulfur hexafluoride microbubbles (Bracco Suisse SA, Switzerland) dissolved in $0.9 \% \mathrm{NaCl}$ with $2 \mu \mathrm{g}$ plasmid in $300 \mu \mathrm{l}$ serum-free DMEM and irradiated with ultrasound [2.0 MHz, 0.37 (mechanical parameter)] for $20 \mathrm{sec}$. At $24 \mathrm{~h}$ post transfection, the cells were cultured with complete medium containing $10 \%$ FBS for an additional $48 \mathrm{~h}$.

Reverse transcription-quantitative PCR (RT-qPCR). Total RNA was extracted from HepG2 cells transfected with PXC9-puro-NC or PXC9-puro-KO using an RNA Extraction kit (BioTeke Corporation) according to the manufacturer's protocol. RNA was reverse transcribed into cDNA using a Reverse Transcriptase M-MLV kit (FSQ-101; Toyobo Life Science) with the volume of $20 \mu \mathrm{l}$ containing $1 \mu \mathrm{l}$ M-MLV, $1 \mu \mathrm{l}$ oligonucleotide $\mathrm{T}, 2 \mu \mathrm{g}$ RNA template and $4 \mu \mathrm{l} 5 \mathrm{x}$ RT buffer at $37^{\circ} \mathrm{C}$ for $15 \mathrm{~min}$ and then $95^{\circ} \mathrm{C}$ for $5 \mathrm{~min}$. RT-qPCR was performed using a SYBR-Green PCR mix (Toyobo Life Science) and specific primers. The sequences of the primers were as follows: Cyclin D1 forward, 5'-CAATGACCCCGC ACGATTTC-3' and reverse, 5'-GGCAGTCCGGGTCAC ACT-3'; cyclin D2 forward, 5'-GGGAAGTTGAAGTGG AACCTG-3' and reverse, 5'-GATCATCGACGGTGGGTA CAT-3'; $\beta$-actin forward, 5'-TGGCACCAGCACAATGAA-3' and reverse, 5'-CTAAGTCATAGTCCGCCTAGAAGCA-3'. The qPCR were performed in triplicate as follows: $94^{\circ} \mathrm{C}$ for $2 \mathrm{~min}$; and 40 cycles of $94^{\circ} \mathrm{C}$ for $15 \mathrm{sec}, 60^{\circ} \mathrm{C}$ for $20 \mathrm{sec}$, and $72^{\circ} \mathrm{C}$ for $20 \mathrm{sec}$. The data were analyzed using the $2^{-\Delta \Delta \mathrm{Cq}}$ method (25).

Western blot analysis. Cells transfected with PXC9-puro-NC or PXC9-puro-KO were collected and lysed using RIPA lysis buffer with $100 \mathrm{mM}$ phenylmethylsulfonyl fluoride (both Beyotime Institute of Biotechnology). Protein samples (30 $\mu \mathrm{g}$ per lane) were separated using 10\% SDS-PAGE and subsequently transferred onto a polyvinylidene difluoride membrane. The membrane was blocked with 5\% skimmed milk in phosphate-buffered saline (PBS) and then incubated with caprin-1 rabbit polyclonal antibody $(1: 1,000$; cat. no. ab38859; Abcam) and GAPDH rabbit polyclonal antibody $(1: 1,000$; cat. no. 10494-1-AP; ProteinTech Group, Inc.) overnight at $4^{\circ} \mathrm{C}$. After washing three times with TBS-Tween-20, the membrane was incubated with horseradish peroxidase-labeled goat anti rabbit secondary antibody (1:5,000; cat. no. BA1054; Wuhan Boster Biological Technology, Ltd.) for $1 \mathrm{~h}$ at room temperature. After washing three times with TBS-Tween-20, the membrane was incubated with Tanon ${ }^{\mathrm{TM}}$ High-sig ECL western blotting substrate (cat. no. 180-5001) and the signal was measured using CCD (both Tanan Science and Technology Co., Ltd.). The protein bands were quantified with Image-Pro Plus version 6 software (Media Cybernetics).

Cell proliferation assay. HepG2 cells transfected with PXC9-puro-NC or PXC9-puro-KO were seeded in triplicate in 96-well plates at $2 \times 10^{3}$ cells/well. When cells attached to the wall, the absorbance at $450 \mathrm{~nm}$ was determined by an enzyme marker (Thermo Fisher Scientific, Inc.) after cell cultured for $0,24,48$, or $72 \mathrm{~h}$. Cell proliferation was determined using a Cell Counting Kit-8 (Beyotime Institute of Biotechnology) according to the manufacturer's protocol at the indicated time points. The optical density at $450 \mathrm{~nm}$ was measured using a multimode microplate reader (Multiskan GO, Thermo Fisher Scientific, Inc.).

Flow cytometry. Cells transfected with PXC9-puro-NC or PXC9-puro-KO were collected and washed twice with pre-cooled PBS. Subsequently the cells were resuspended in pre-cooled $70 \%$ ethanol and fixed overnight $4^{\circ} \mathrm{C}$. After two washes with PBS, cells were stained with $5 \mu \mathrm{g} / \mathrm{ml}$ propidium iodide (dissolved in PBS with $100 \mu \mathrm{g} / \mathrm{ml}$ RNaseA and $0.2 \%$ Triton X-100) for $30 \mathrm{~min}$ at $4^{\circ} \mathrm{C}$ in darkness. After two washes with PBS, the cells were subjected to flow cytometric analysis using standard procedures. The results were analyzed using the cell cycle fitting software FlowJoLLC version 10 (BD Biosciences).

Cell migration assay. HepG2 cells transfected with PXC9-puro-NC or PXC9-puro-KO were seeded at $5 \times 10^{4}$ cells/well and cultured in triplicate in $200 \mu 1$ serum-free DMEM in the upper chambers of 24-well Transwell chambers (8- $\mu \mathrm{m}$ pores; Corning Inc.). The lower chambers contained $800 \mu \mathrm{l}$ DMEM containing $20 \%$ FBS. After $48 \mathrm{~h}$, the HepG2 cells that had not migrated and remained on the surface membrane of the upper chambers were removed using a cotton swab, and the cells that had migrated to the bottom surface of the upper chambers were fixed with $4 \%$ paraformaldehyde at room temperature for $15 \mathrm{~min}$ and stained with $0.05 \%$ crystal violet for $15 \mathrm{~min}$ at room temperature. Images were captured under a light microscope (MSHOT ML 31, Guangzhou, China) at $\times 400$ magnification. The number of cells that had migrated in 3-5 random fields were counted in a blinded manner.

Wound healing assay. Cells transfected with PXC9-puro-NC or PXC9-puro-KO were seeded in 24-well plates at $5 \times 10^{4}$ cells/well and cultured until the cells reached $100 \%$ confluency. Subsequently the monolayer of cells was wounded using a micropipette tip with a similar width in two groups. The plate was rinsed three times with PBS to wash away the cell debris produced by the scratching. The cells were cultured continuously with DMEM containing 2\% FBS for serum starvation and $4 \mu \mathrm{g} / \mathrm{ml}$ mitomycin (to inhibit cell division to remove the effect of cell proliferation) and images were captured at 0, 6, 24 and $48 \mathrm{~h}$ after scratching. Migration distance was calculated by dividing the scratch area by the width of scratch, which analyzed by with Image-Pro Plus version 6 software (Media Cybernetics).

Statistical analysis. Statistical analysis was performed using SPSS version 22.0 software (IBM Corp.). The differences between the two groups were compared using Student's t-tests. The differences among three groups were compared by one-way ANOVA with LSD post-hoc test. The results are expressed as mean \pm standard deviation. All experiments were repeated three times. Pearson's $\chi^{2}$ test and a Fisher's exact test were used to analyze the association between caprin-1 protein expression levels and clinicopathological features in the liver cancer cases. Overall survival was analyzed using the Kaplan-Meier test and differences were assessed using a log-rank test. Univariate analysis and multivariate survival analysis were performed using the Cox proportional hazard regression model. The relative risks of death are presented as adjusted hazard ratios (HRs) 
Table I. Association of caprin-1 protein and mRNA expression levels with clinicopathological characteristics in patients with liver cancer.

\begin{tabular}{|c|c|c|c|c|c|c|c|}
\hline \multirow[b]{2}{*}{ Clinical features } & \multicolumn{4}{|c|}{ Tissue microarray } & \multicolumn{3}{|c|}{ The Cancer Genome Atlas database } \\
\hline & Number & Low, n (\%) & High, n (\%) & P-value & Number & Mean \pm SD & P-value \\
\hline Cancer & 39 & $7(17.9)$ & $32(82.1)$ & $<0.001^{\mathrm{a}}$ & 134 & $3,516.17 \pm 1,370.19$ & - \\
\hline Benign & 30 & $24(80.0)$ & $6(20.0)$ & & - & - & - \\
\hline \multicolumn{8}{|l|}{ Age, years } \\
\hline$<45$ & 15 & $2(13.3)$ & $13(86.7)$ & 0.444 & $67(<65)$ & $3,300.31 \pm 1,319.87$ & $0.034^{\mathrm{b}}$ \\
\hline$\leq 45$ & 24 & $5(20.8)$ & $19(79.2)$ & & $67(\geq 65)$ & $3,784.58 \pm 1,299.38$ & \\
\hline \multicolumn{8}{|l|}{ Sex } \\
\hline Male & 8 & $1(12.5)$ & $7(87.5)$ & 0.554 & 80 & $3,407.94 \pm 1,228.09$ & 0.154 \\
\hline Female & 31 & $6(19.4)$ & $25(80.6)$ & & 54 & $3,741.71 \pm 1,450.62$ & \\
\hline \multicolumn{8}{|l|}{ Pathological grade } \\
\hline$\leq 2$ & 29 & $3(10.3)$ & $26(89.7)$ & 0.131 & - & - & - \\
\hline$>2$ & 9 & $3(33.3)$ & $6(66.7)$ & - & - & - & - \\
\hline \multicolumn{8}{|l|}{ Clinical stage } \\
\hline $\mathrm{I}-\mathrm{II}$ & 15 & $7(46.7)$ & $8(53.3)$ & $<0.001^{\mathrm{a}}$ & - & - & - \\
\hline III-IV & 24 & $0(0.0)$ & $24(100.0)$ & & - & - & - \\
\hline \multicolumn{8}{|l|}{ Tumor invasion } \\
\hline $\mathrm{T} 1-\mathrm{T} 2$ & 15 & $7(46.7)$ & $8(53.3)$ & $<0.001^{\mathrm{a}}$ & 86 & $3,473.89 \pm 1,067.72$ & $0.044^{\mathrm{b}}$ \\
\hline $\mathrm{T} 3-\mathrm{T} 4$ & 24 & $0(0.0)$ & $24(100.0)$ & & 48 & $3,865.27 \pm 1,079.35$ & \\
\hline \multicolumn{8}{|c|}{ Lymph node metastasis ${ }^{\mathrm{c}}$} \\
\hline No & - & - & - & - & 83 & $3,649.43 \pm 1,407.77$ & 0.902 \\
\hline N1 & - & - & - & - & 3 & $3,751.22 \pm 1,195.26$ & \\
\hline \multicolumn{8}{|c|}{ Distant metastasis $^{\mathrm{d}}$} \\
\hline M0 & - & - & - & - & 99 & $3,593.43 \pm 1,401.42$ & 0.534 \\
\hline M1 & - & - & - & - & 3 & $3,084.15 \pm 725.15$ & \\
\hline
\end{tabular}

${ }^{\mathrm{a}} \mathrm{P}<0.001,{ }^{\mathrm{b}} \mathrm{P}<0.05$. ${ }^{\mathrm{c}}$ In total, 48 patients had no information of lymph node metastasis. ${ }^{\mathrm{d} I n}$ total, 32 patients had no information regarding distant metastasis. Low, <mean caprin-1 protein expression level; High, > mean caprin-1 protein expression level.

and $95 \%$ confidence intervals (CIs). $\mathrm{P}<0.05$ was considered to indicate a statistically significant difference.

\section{Results}

Caprin-1 is highly expressed in liver cancer tissue. Detailed information on the clinical characteristics of all patients in the present study are shown in Table I. As shown in Fig. 1, caprin-1 expression was primarily localized to the cytoplasm of liver cancer cells with high expression levels, whereas caprin-1expression levels were weak in normal liver tissue. Of the 39 liver cancer samples, 7 samples (17.9\%) showed low expression levels of caprin-1 (<mean expression levels of caprin-1), whereas 32 samples $(82.1 \%)$ showed strong staining with high expression levels ( $>$ mean expression levels of caprin-1). In addition, the expression levels of caprin-1 in liver cancer tissues were significantly higher compared with that in normal liver tissues (IRS, 9.44 \pm 2.62 in liver cancer vs. $5.60 \pm 1.65$ in normal tissue; $\mathrm{P}<0.001$; Fig. 1B).

Association between caprin-1 expression and clinical characteristics of patients. When analyzing the relationship between caprin-1 and clinical characteristics of the patients using TMA data, the results revealed that caprin-1 protein overexpression was associated with advanced clinical stage and tumor invasion (both $\mathrm{P}<0.001$ ) and enhanced tumor invasion $(\mathrm{P}<0.001)$. However, high caprin-1 levels were not associated with age or sex (both $\mathrm{P}>0.05$; Table I). The results from the TCGA database, which contained high-throughput sequencing data for caprin-1 mRNA expression in 154 liver cancer tissues, showed that caprin-1 mRNA expression was upregulated in older patients $(\mathrm{P}=0.034)$ and tumor-infiltrating liver cancer tissues $(\mathrm{P}=0.044$; Table I).

Caprin-1 expression and prognosis of liver cancer from TCGA data. The association between caprin-1 expression levels and survival time of patients with liver cancer was analyzed using the Kaplan-Meier method from data downloaded from TCGA database. As shown in Fig. 2, the overall survival time and disease-free survival time of patients with liver cancer with high caprin-1 expression levels were significantly shorter compared with that in patients with liver cancer with low caprin- 1 expression levels ( $\mathrm{P}=0.002$ and $\mathrm{P}=0.033$, respectively). In patients without metastatic disease, the metastasis-free 


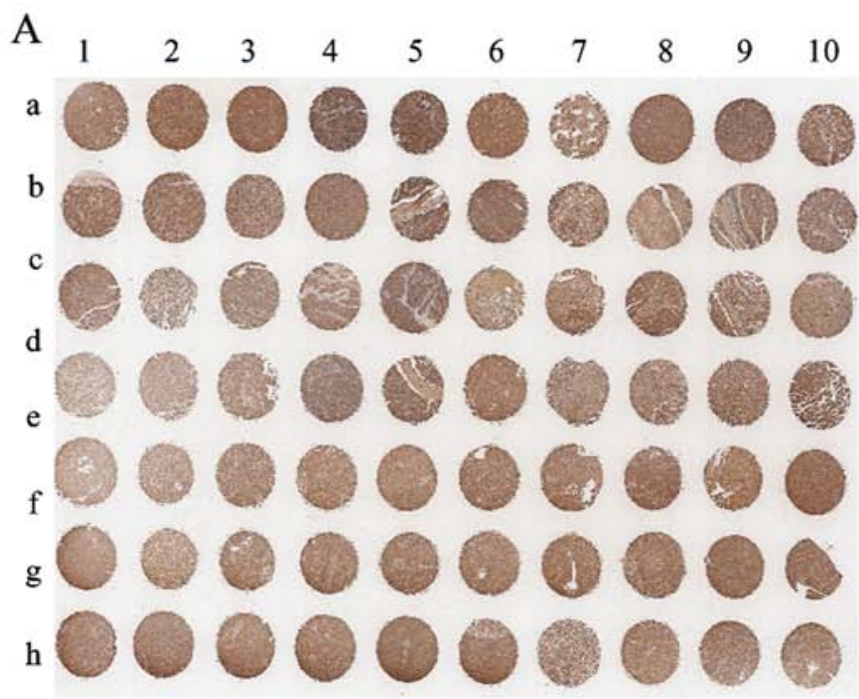

B

$\mathrm{C}$

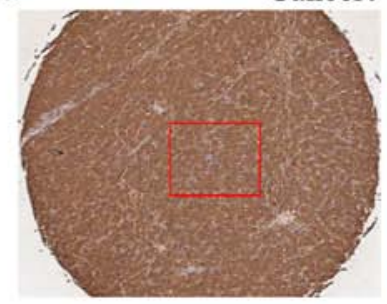

E

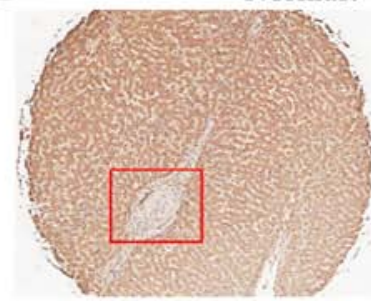

strongly

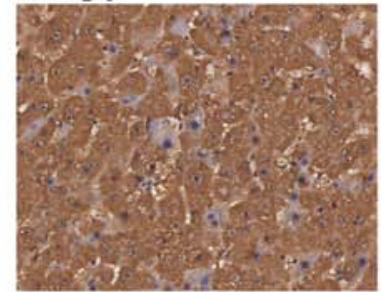

mediately

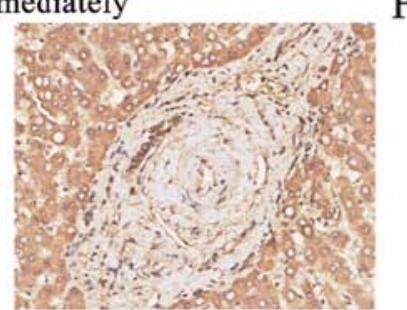

D

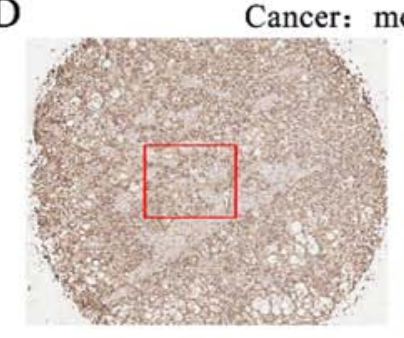

F

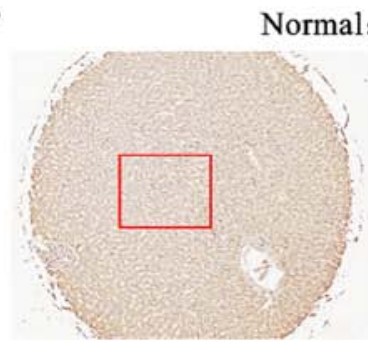

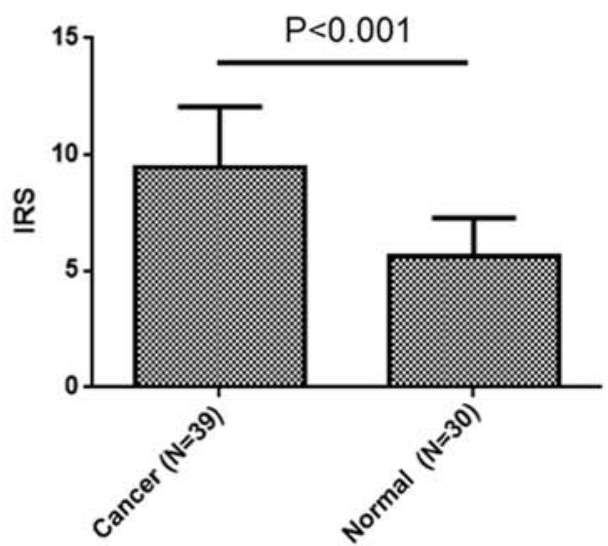

Cancer: mediately

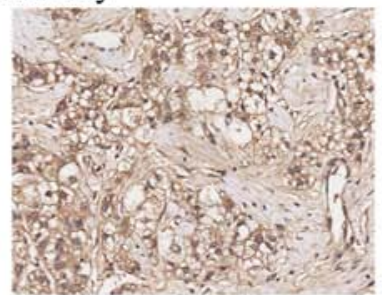

weakly

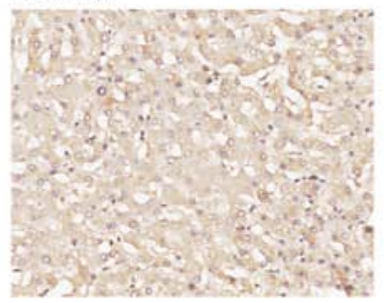

Figure 1. Protein expression of caprin-1 in liver cancer. (A) The expression levels of caprin-1 in the TMA, containing 40 liver cancer tissues, 10 peritumoral tissues and 20 normal tissues. (B) Statistical analysis demonstrating a higher IRS in tumor tissues compared with normal tissues. (C-F) Representative images of cancer tissue and normal tissue at different staining intensity and at different magnifications. IRS, immune response score; TMA, tissue microarray.

and disease-free survival rates of patients with high caprin-1 expression levels were significantly lower compared with that in patients with low caprin-1 expression levels $(\mathrm{P}=0.003$ and $\mathrm{P}=0.033$, respectively).

Caprin-1 is an independent predictor for liver cancer survival. Univariate analysis showed that besides person neoplasm status (HR, 1.826; 95\% CI, 1.060-3.146; $\mathrm{P}=0.030)$ and tumor invasion (HR, 2.701; 95\% CI, 1.203-6.061; $\mathrm{P}=0.016$ ), caprin-1 expression was also a significant prognostic factor for disease-free survival in patients with liver cancer (HR, 2.284; 95\% CI, 1.328-3.930; $\mathrm{P}=0.003$; Table II). Multivariate analysis using the Cox proportional hazard model showed that besides person neoplasm status (HR,3.573; 95\% CI,1.421-8.938; $\mathrm{P}=0.007)$, sex (HR, 3.647; 95\% CI,1.493-8.909; $\mathrm{P}=0.005)$, tumor invasion(HR, 52.503; 95\% CI, 6.706-411.079; $\mathrm{P}<0.001)$, and distant metastasis (HR, 0.048; 95\% CI, 0.004-0.574; $\mathrm{P}=0.048$ ), high caprin-1 expression was also a significant independent prognostic factor in patients with liver cancer (HR, 7.299; 95\% CI, 2.614-20.379; P<0.001; Table II).
Caprin-1 knockdown inhibits cell proliferation by arresting cells at $G_{0} / G_{l}$ phase. Next, the underlying mechanism of caprin-1 in liver cancer was studied in human HepG2 liver cancer cells. A knockout plasmid for caprin-1 was constructed to decrease the expression levels of caprin-1 in cultured cells. As shown in Fig. 3A and B, after HepG2 cells were transfected with either PXC9-puro-KO-1, PXC9-puro-KO-2 or PXC9-puro-NC, the expression levels of caprin-1 were significantly decreased, by $52 \%$ in cells treated with PXC9-puro-KO-1 and $67 \%$ in cells treated with PXC9-puro-KO-2. PXC9-puro-KO-2 was used for further experiments because of its higher knockout efficiency. Downregulation of caprin-1 significantly inhibited the proliferation of HepG2 cells (Fig. 3C) and the cells were arrested in $G_{0} / G_{1}$ phase (Fig. 3D and $E$ ). The mRNA levels of cyclin D1 and D2 were both downregulated in caprin-1 KO cells (Fig. 3F).

Caprin-1 knockdown inhibits cell migration. The effect of caprin-1 knockdown on cell invasion was also analyzed. As shown in Figs. 4 and 5, after HepG2 cells were transfected 
A

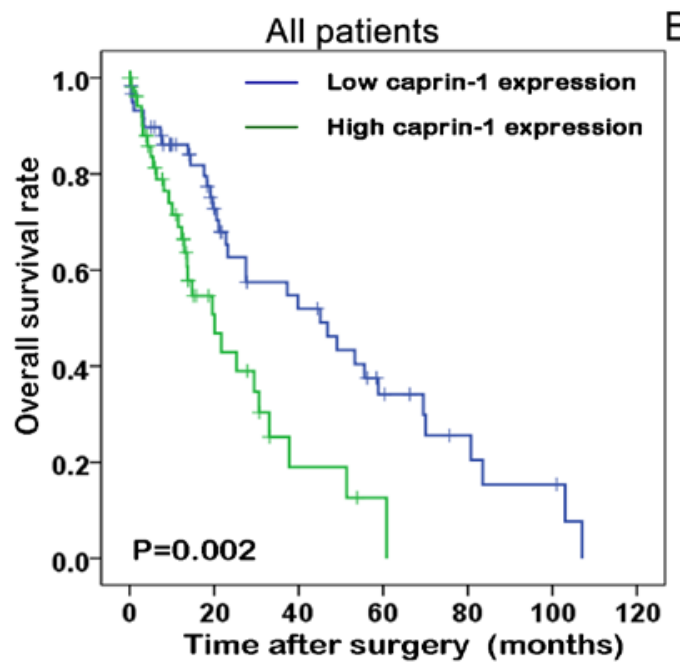

C

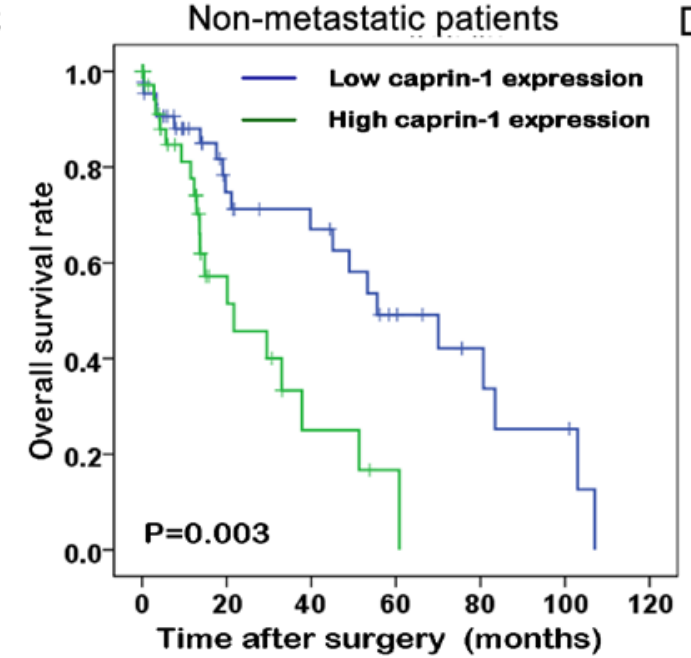

B

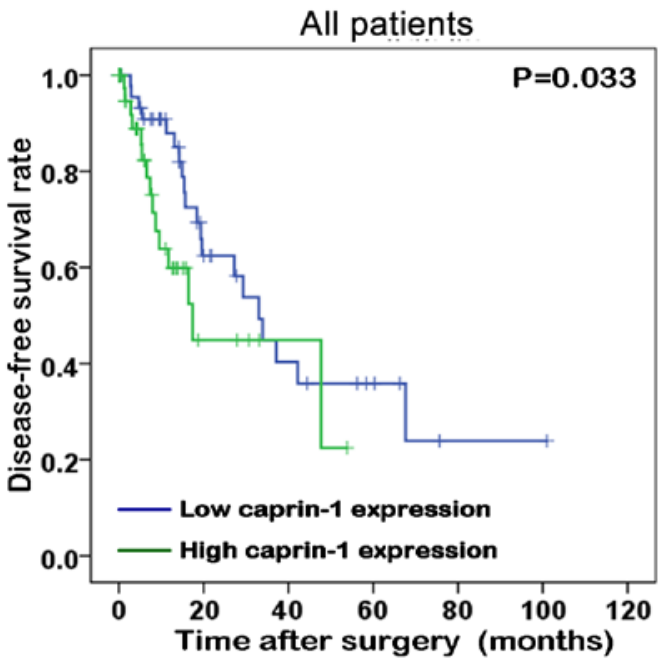

D

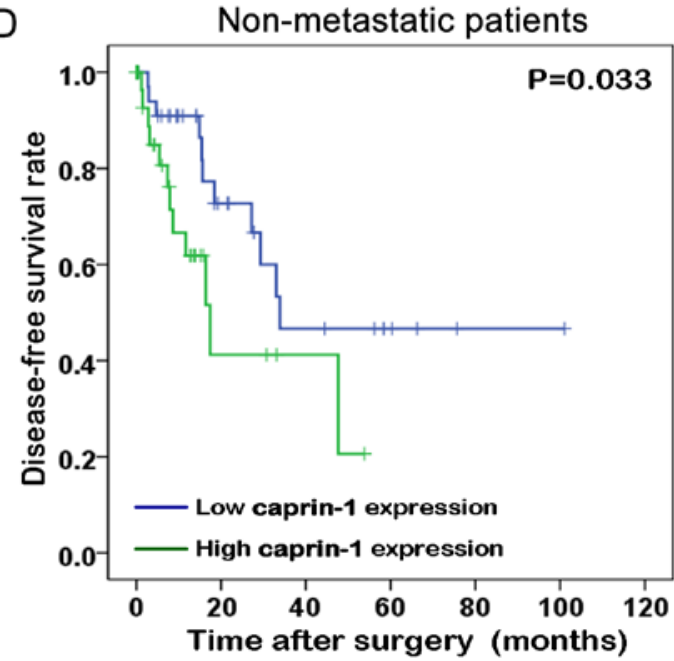

Figure 2. Kaplan-Meier survival analysis of caprin-1 expression levels in patients with liver cancer collected from The Cancer Genome Atlas database. (A) Overall survival rate of all patients. (B) Disease-free survival rate of all patients. (C) Overall survival rate of patients without metastasis. (D) Disease-free survival rate of patients without metastasis.

with either PXC9-puro-KO-2 or PXC9-puro-NC, the migration ability of HepG2 cells were significantly inhibited in the cells transfected with PXC9-puro-KO-2, indicating that downregulation of caprin-1 inhibited the migration of HepG2 cells.

\section{Discussion}

In the present study, caprin-1 expression levels in liver cancer tissues and HepG2 cells were significantly higher compared with that in normal liver tissues and cells. Higher expression levels of caprin-1 were associated with advanced stage and enhanced tumor invasion, independent of age or sex. Using TCGA data, it was found that the overall survival time and disease-free survival time of patients with high expression levels of caprin-1 were significantly shorter compared with that in patients with low expression levels. Multivariate analysis showed that high expression levels of caprin-1 were a significant independent prognostic factor in patients with liver cancer. The aforementioned results suggested that the expression levels of caprin-1 are associated with the occurrence, development and prognosis of liver cancer. Further experiments in HepG2 cells showed that knockdown of caprin-1 downregulated the expression of cyclin D1 and D2, and inhibited cell proliferation as well as cell migration.

Caprin-1 consists of 709 amino acids and contains an RGG sequence unique to RNA-binding proteins (16). Lack of caprin-1 may delay $G_{1}$ to $S$ phase progression within the cell cycle (17). Sabile et al (21) found that caprin-1 overexpression can significantly promote the growth and lung metastasis of mouse osteosarcoma. The present study showed that high caprin-1 expression levels were associated with specific advanced clinicopathological features in liver cancer, which is in accordance with the growth promotion function of caprin-1 in osteosarcoma (21). Together, these studies suggest that caprin-1 serves an important role in the regulation of malignant tumors.

Next, the mechanism underlying the association of caprin-1 and liver cancer prognosis was explored. The present study showed that knockdown of caprin-1 inhibited proliferation by arresting cells at $G_{0} / G_{1}$ phase and downregulated the expression of cyclin D1 and D2. Cyclin D1 and D2 are proteins that couple with extracellular signals to the biochemical machinery 
Table II. Prognostic value of caprin-1 mRNA expression levels for overall survival based on Cox proportional hazards model.

A, Univariate analysis

Overall survival

Variable

HR $(95 \% \mathrm{CI})$

P-value

Tumor status, tumor vs. tumor free

$1.826(1.060-3.146)$

$0.030^{\mathrm{a}}$

Age, $\geq 65$ vs. $<65$ years

$0.768(0.463-1.276)$

0.309

Sex, male vs. female

$1.312(0.782-2.201)$

0.304

Clinical stage, I-II vs. III-IV

$1.120(0.646-1.943)$

0.686

Tumor invasion, T1-T2 vs. T3-T4

$2.701(1.203-6.061)$

$0.016^{\mathrm{a}}$

Lymph node stage, N0 vs. N1

$0.676(0.091-5.005)$

0.676

Distant metastasis, M0 vs. M1

$2.917(0.879-9.674)$

0.080

Caprin-1 expression, low vs. high

$2.284(1.328-3.930)$

$0.003^{\mathrm{b}}$

B, Multivariate analysis

Overall survival

Variable

HR $(95 \% \mathrm{CI})$

P-value

Personal neoplasm status, with tumor vs. tumor free

$3.573(1.421-8.938)$

$0.007^{\mathrm{b}}$

Age, $\geq 65$ vs. $<65$ years

$2.208(0.934-5.218)$

0.071

Sex, male vs. female

$3.647(1.493-8.909)$

$0.005^{\mathrm{b}}$

Clinical stage, I-II vs. III-IV

$0.877(0.369-2.084)$

0.766

Tumor invasion, T1-T2 vs. T3-T4

$52.503(6.706-411.079)$

$<0.001^{\mathrm{c}}$

Lymph node stage, N0 vs. N1

0.407 (0.046-3.646)

0.422

Distant metastasis, M0 vs. M1

$0.048(0.004-0.574)$

$0.048^{\mathrm{a}}$

Caprin-1 expression, low vs. high

7.299 (2.614-20.379)

$<0.001^{\mathrm{c}}$

${ }^{\mathrm{a}} \mathrm{P}<0.05,{ }^{\mathrm{b}} \mathrm{P}<0.01,{ }^{\mathrm{c}} \mathrm{P}<0.001$. HR, hazard ratio; $\mathrm{CI}$, confidence interval; Low, $<$ mean caprin-1 protein expression level; High, $>$ mean caprin-1 protein expression level.

governing progression through $\mathrm{G}_{1}$ phase of the mammalian cell division cycle (26). These proteins are frequently deregulated in cancer and are biomarkers of cancer phenotype and disease progression (27). The data of the present study also showed that caprin-1 knockdown inhibited the migration of HepG2 cells. Thus, downregulation of caprin-1 expression inhibited proliferation and migration in tumor cells and was associated with poor prognosis in patients with liver cancer. This is consistent with previous reports; for example, caprin-1 was reported to be essential for the proliferation of B lymphocyte cells (17). In addition, in osteosarcoma cells, caprin-1 overexpression can activate the MAPK/ERK and PI3K/Akt signaling pathways, which are involved in the regulation of cell proliferation, apoptosis and survival (21). Overexpression of caprin-1 promotes the proliferation and invasion of breast cancer cells and microRNA (miR)-223 inhibits cancer cell proliferation by targeting the 3'untranslated region of caprin-1; thus, downregulation of caprin-1 expression can inhibit cancer cell proliferation and invasion (22). miR-193a, which is highly expressed in colon tumor cells, causes cell cycle $G_{1}$ arrest and inhibits cell proliferation by inhibiting Caprin-1 expression (23). The effect of miR-193a-mediated disruption of the caprin-1/G3BP-1/c-MYC/Cyclin D2 complex may be a potential target for anticancer therapeutic applications (23). The data of the present study indicates that caprin-1 can regulate the development of liver cancer through its effects on cell proliferation and migration.

In a previous study, the predictive value of caprin-1 in liver cancer was reported (28). It was found that upregulation of caprin-1 expression is associated with poor prognosis in liver cancer, which is consistent with the conclusion of the present study. However, the relationship between caprin-1 and metastasis in the previous study (28) was not investigated, although both lymph node metastasis and distant metastasis showed no difference with the level of caprin-1 expression. Moreover, the present study explored the underlying mechanism, demonstrating that the predictive value of caprin-1 for the prognosis of liver cancer may be associated with its function in cancer cell proliferation and migration.

The present study had several limitations. First, the TMA cancer tissue and the control tissue samples were not all compared or were they from the same patients in the present study, and only 10 adjacent samples were collected, which may affect the comparison of caprin-1 expression between 
A

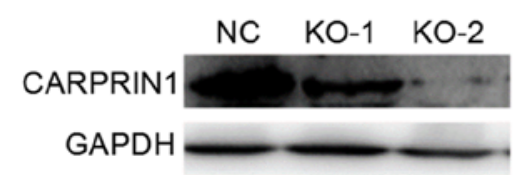

B

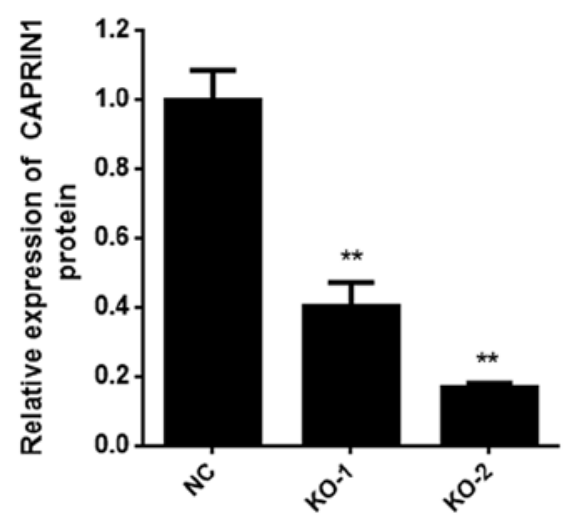

C

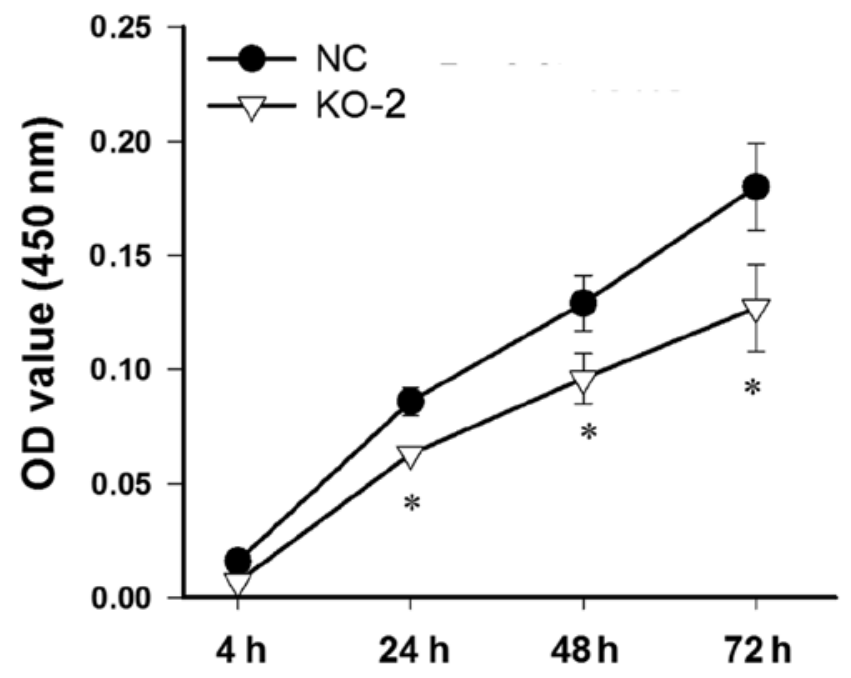

D

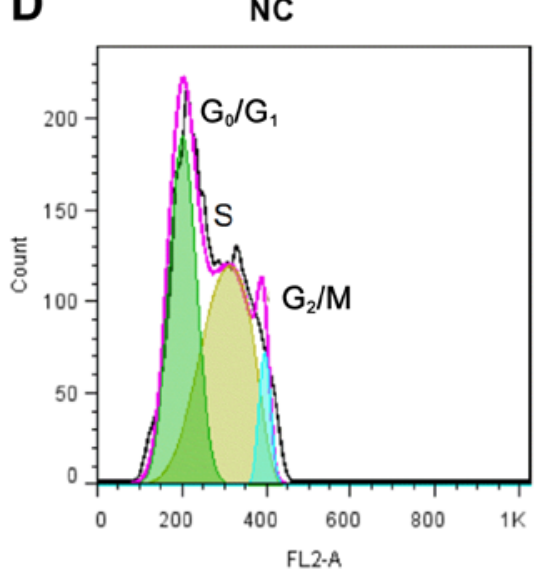

$\mathbf{F}$

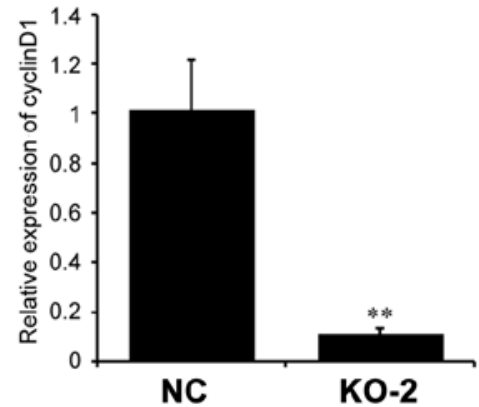

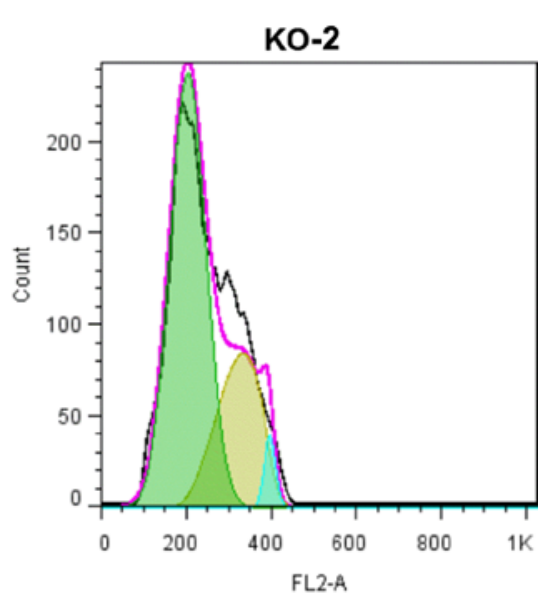

E

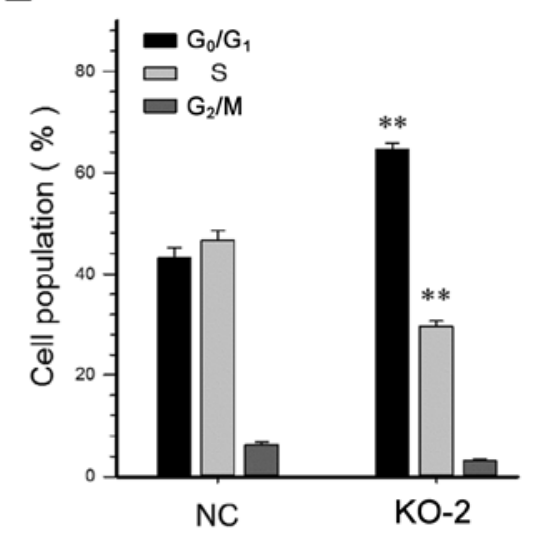

Figure 3. Knockdown of caprin-1 inhibits the proliferation and apoptosis of HepG2 cells. HepG2 cells transfected with PXC9-puro-NC, PXC9-puro-KO-1 or -2 were seeded in triplicate in 96 -well plates at $2 \times 10^{3}$ cells/well. (A) Western blot analysis and (B) quantitative analysis of caprin-1 protein expression in cells transfected with PXC9-puro-NC, PXC9-puro-KO-1 or PXC9-puro-KO-2 determined at $48 \mathrm{~h}$ post-transfection. Cells transfected with PXC9-puro-KO-2 showed lower expression and were selected for later experiments. (C) Statistical analysis of proliferation rates of HepG2 cells transfected with PXC9-puro-NC and PXC9-puro-KO-2. (D) Flow cytometry of cell cycle and (E) quantitative analysis of the results at $48 \mathrm{~h}$ post-transfection. (F) mRNA levels of cyclin D1 and cyclin D2 were determined using reverse transcription-quantitative PCR 48 h post-transfection. ${ }^{*} \mathrm{P}<0.05$ or ${ }^{* *} \mathrm{P}<0.01$ vs. respective NC. NC, negative control; KO, knockout; OD, optical density.

two groups due to possible genetic differences between cancer tissues and those un-paired samples. However, the present data are consistent with the findings of a previous report (28), indicating that the impact of no paired samples on the results was small. Secondly, a preliminary study was conducted to investigate the mechanism of caprin-1 in liver cancer and further research is required to investigate the underlying molecular mechanisms.

In conclusion, the present study showed that caprin-1 is overexpressed in liver cancer tissues and is associated with the clinicopathological features of liver cancer. Moreover, high caprin-1 expression levels are associated with poor prognosis 


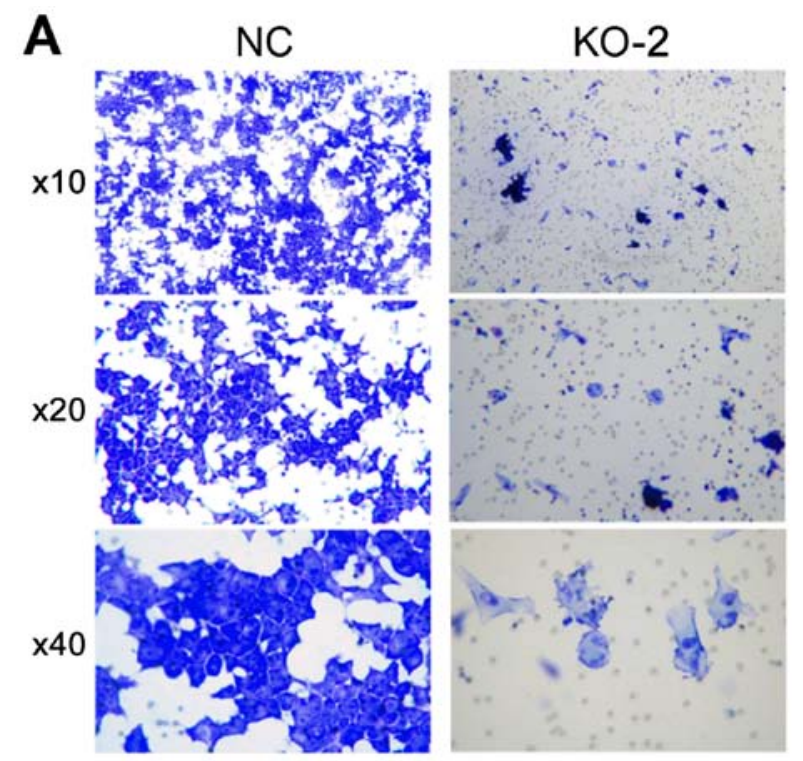

B

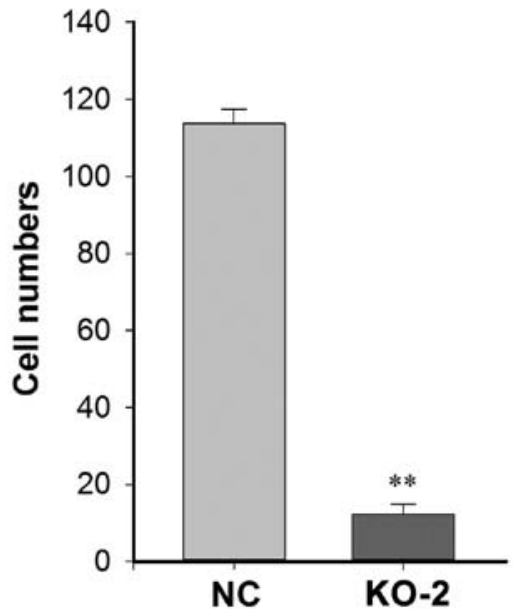

Figure 4. Knockdown of caprin-1 inhibits the migration of HepG2 cells. HepG2 cells transfected with PXC9-puro-NC or PXC9-puro-KO were seeded at $5 \times 10^{4}$ cells/well in the upper chambers of 24 -well Transwell chambers. After $48 \mathrm{~h}$, the cells that had migrated to the bottom surface of the upper chambers were fixed and stained with $0.05 \%$ crystal violet. (A) Representative invasion images at different magnifications. (B) Quantitative analysis of migration of HepG2 cells. Experiments were repeated three times. ${ }^{* *} \mathrm{P}<0.01$ vs. NC. NC, negative control; KO, knockout.

A
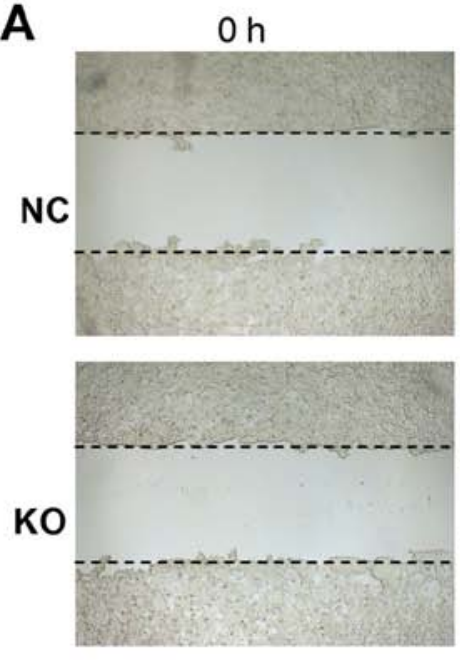

B
$6 \mathrm{~h}$
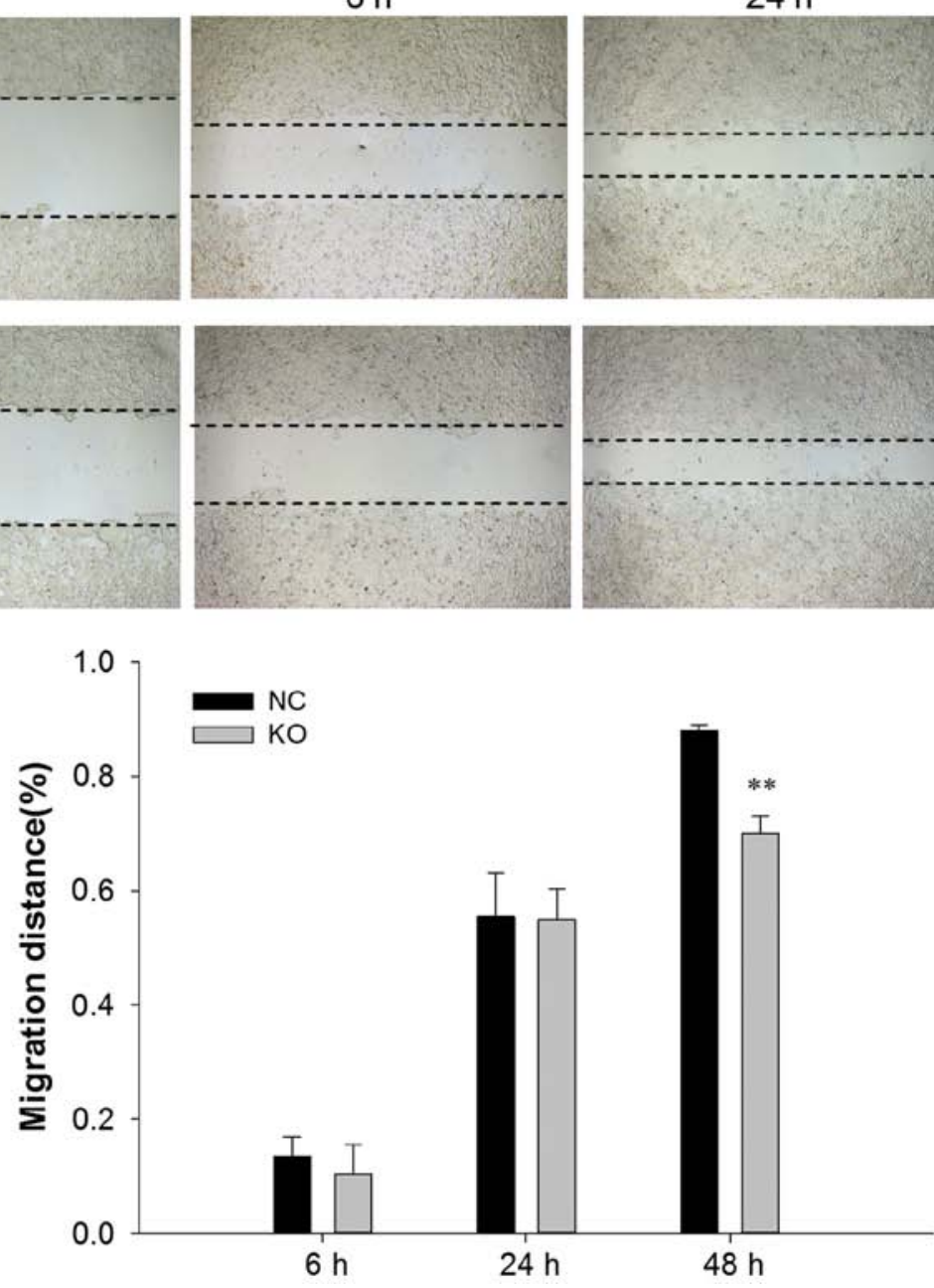

$24 \mathrm{~h}$
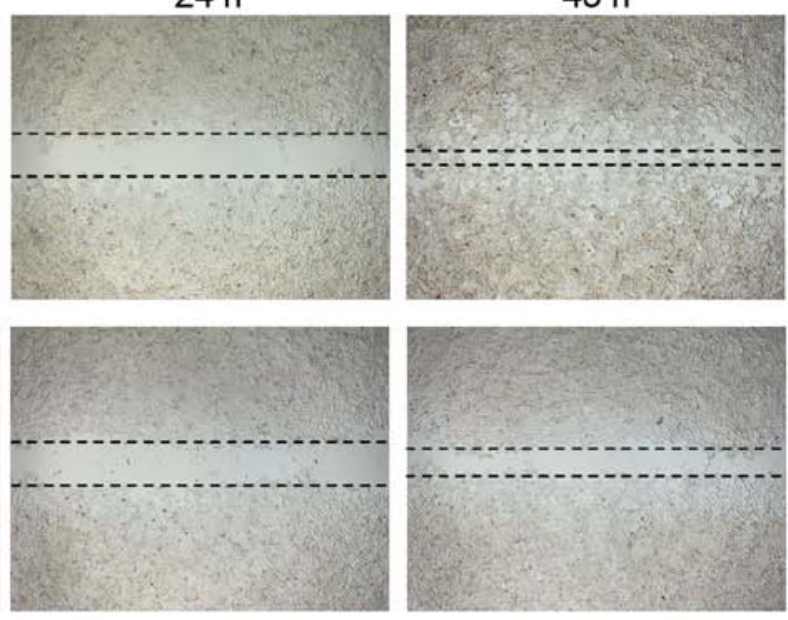

$48 \mathrm{~h}$

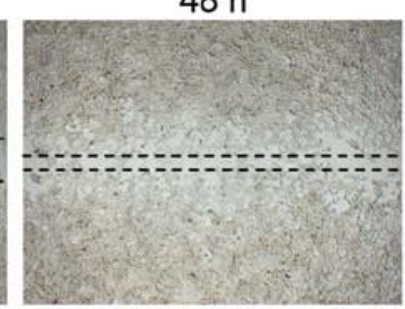


in liver cancer. Caprin-1 knockdown inhibits the proliferation and migration of liver cancer cells. Caprin-1 may serve as a novel prognostic biomarker for liver cancer, assisting in liver cancer treatment and prediction of patient prognosis.

\section{Acknowledgements}

Not applicable.

\section{Funding}

This study was supported by Science and Technology Planning Project of Guangdong Province, China (grant no. 2016A020215015).

\section{Availability of data and materials}

The datasets generated and/or analyzed during the present study are available from the corresponding author on reasonable request.

\section{Authors' contributions}

XMG, JCS, FFZ and LWP conceived and designed the study. XMG, FFZ, LWP and JLC collected data and conducted the research. JLC, JCL and HXW analyzed and interpreted the data. FFZ and JLC wrote the initial manuscript. XMG and JCS revised the manuscript. XMG had primary responsibility for final content. All authors read and approved the final manuscript.

\section{Ethics approval and consent to participate}

The company that provided the clinical samples had no individual Ethics Committee, thus the present study was approved by the Ethics Committee of Guangzhou Red Cross Hospital (Guangzhou, China; approval no. 2017-047-01). All procedures performed in studies involving human participants were in accordance with the ethics standards of the institutional and national research committee and with the 1964 Declaration of Helsinki and its later amendments or comparable ethics standards. Written informed consent was provided by all individual participants included in the present study.

\section{Patient consent for publication}

Not applicable.

\section{Competing interests}

The authors declare that they have no competing interests.

\section{References}

1. Global Burden of Disease Cancer Collaboration; Fitzmaurice C, Dicker D, Pain A, Hamavid H, Moradi-Lakeh M, MacIntyre MF, Allen C, Hansen G, Woodbrook R, et al: The global burden of cancer 2013. JAMA Oncol 1: 505-527, 2015.

2. Ferlay J, Soerjomataram I, Dikshit R, Eser S, Mathers C, Rebelo M, Parkin DM, Forman D and Bray F: Cancer incidence and mortality worldwide: Sources, methods and major patterns in GLOBOCAN 2012. Int J Cancer 136: E359-E386, 2015.
3. Torre LA, Bray F, Siegel RL, Ferlay J, Lortet-Tieulent J and Jemal A: Global cancer statistics, 2012. CA Cancer J Clin 65: 87-108, 2015

4. GBD 2013 Mortality and Causes of Death Collaborators: Global, regional, and national age-sex specific all-cause and cause-specific mortality for 240 causes of death, 1990-2013: A systematic analysis for the Global Burden of Disease Study 2013. Lancet 385: 117-171, 2015.

5. Daher S, Massarwa M, Benson AA and Khoury T: Current and future treatment of hepatocellular carcinoma: An updated comprehensive review. J Clin Transl Hepatol 6: 69-78, 2018.

6. Llovet JM and Bruix J: Early diagnosis and treatment of hepatocellular carcinoma. Baillieres Best Pract Res Clin Gastroenterol 14: 991-1008, 2000.

7. Huang TS, Shyu YC, Turner R, Chen HY and Chen PJ: Diagnostic performance of alpha-fetoprotein, lens culinaris agglutinin-reactive alpha-fetoprotein, des-gamma carboxyprothrombin, and glypican-3 for the detection of hepatocellular carcinoma: A systematic review and meta-analysis protocol. Syst Rev 2: 37, 2013.

8. Ioannou GN, Perkins JD and Carithers RL Jr: Liver transplantation for hepatocellular carcinoma: Impact of the MELD allocation system and predictors of survival. Gastroenterology 134: 1342-1351, 2008

9. Li J, Gao JZ, Du JL and Wei LX: Prognostic and clinicopathological significance of glypican-3 overexpression in hepatocellular carcinoma: A meta-analysis. World J Gastroenterol 20: 6336-6344, 2014.

10. Wang Y, Peng C, Cheng Z, Wang X, Wu L, Li J, Huang C, Guo Q and Cai $\mathrm{H}$ : The prognostic significance of preoperative neutrophil-lymphocyte ratio in patients with hepatocellular carcinoma receiving hepatectomy: A systematic review and meta-analysis. Int J Surg 55: 73-80, 2018.

11. Zhou Y, Sui C, Li B, Yin Z, Tan Y, Yang J and Liu Z: Repeat hepatectomy for recurrent hepatocellular carcinoma: A local experience and a systematic review. World J Surg Oncol 8: 55, 2010.

12. Ludwig JA and Weinstein JN: Biomarkers in cancer staging, prognosis and treatment selection. Nat Rev Cancer 5: 845-856, 2005.

13. Zhang J, Zhang M, Ma H, Song X, He L, Ye X and Li X: Overexpression of glypican-3 is a predictor of poor prognosis in hepatocellular carcinoma: An updated meta-analysis. Medicine (Baltimore) 97: e11130, 2018

14. Wang Q, Wang G, Liu C and He X: Prognostic value of CpG island methylator phenotype among hepatocellular carcinoma patients: A systematic review and meta-analysis. Int J Surg 54: 92-99, 2018.

15. Zheng J, Cai J, Li H, Zeng K, He L, Fu H, Zhang J, Chen L, Yao J, Zhang Y, et al: Neutrophil to lymphocyte ratio and platelet to lymphocyte ratio as prognostic predictors for hepatocellular carcinoma patients with various treatments: A meta-analysis and systematic review. Cell Physiol Biochem 44: 967-981, 2017.

16. Grill B, Wilson GM, Zhang KX, Wang B, Doyonnas R, Quadroni $M$ and Schrader JW: Activation/division of lymphocytes results in increased levels of cytoplasmic activation/proliferation-associated protein-1: Prototype of a new family of proteins. J Immunol 172: 2389-2400, 2004.

17. Wang B, David MD and Schrader JW: Absence of caprin-1 results in defects in cellular proliferation. J Immunol 175: 4274-4282, 2005.

18. Solomon S, Xu Y, Wang B, David MD, Schubert P, Kennedy D and Schrader JW: Distinct structural features of caprin-1 mediate its interaction with G3BP-1 and its induction of phosphorylation of eukaryotic translation initiation factor 2 alpha, entry to cytoplasmic stress granules, and selective interaction with a subset of mRNAs. Mol Cell Biol 27: 2324-2342, 2007.

19. Kedersha N, Panas MD, Achorn CA, Lyons S, Tisdale S, Hickman T, Thomas M, Lieberman J, McInerney GM, Ivanov P, et al: G3BP-Caprin1-USP10 complexes mediate stress granule condensation and associate with $40 \mathrm{~S}$ subunits. J Cell Biol 212: 845-860, 2016.

20. Copsey AC, Cooper S, Parker R, Lineham E, Lapworth C, Jallad D, Sweet S and Morley SJ: The helicase, DDX3X, interacts with poly(A)-binding protein 1 (PABP1) and caprin-1 at the leading edge of migrating fibroblasts and is required for efficient cell spreading. Biochem J 474: 3109-3120, 2017.

21. Sabile AA, Arlt MJ, Muff R, Husmann K, Hess D, Bertz J, Langsam B, Aemisegger C, Ziegler U, Born W, et al: Caprin-1, a novel Cyr61-interacting protein, promotes osteosarcoma tumor growth and lung metastasis in mice. Biochim Biophys Acta 1832: 1173-1182, 2013. 
22. Gong B, Hu H, Chen J, Cao S, Yu J, Xue J, Chen F, Cai Y, He H and Zhang L: Caprin-1 is a novel microRNA-223 target for regulating the proliferation and invasion of human breast cancer cells. Biomed Pharmacother 67: 629-636, 2013.

23. Teng Y, Ren Y, Hu X, Mu J, Samykutty A, Zhuang X, Deng Z, Kumar A, Zhang L, Merchant ML, et al: MVP-mediated exosomal sorting of miR-193a promotes colon cancer progression. Nat Commun 8: 14448, 2017.

24. Edge SB: American Joint Committee on Cancer: AJCC cancer staging manual. 7th edition. New York, Springer, 2010.

25. Livak KJ and Schmittgen TD: Analysis of relative gene expression data using real-time quantitative PCR and the 2(-Delta Delta C(T)) method. Methods 25: 402-408, 2001.

26. Sherr CJ: D-type cyclins. Trends Biochem Sci 20: 187-190, 1995.
27. Musgrove EA, Caldon CE, Barraclough J, Stone A and Sutherland RL: Cyclin D as a therapeutic target in cancer. Nat Rev Cancer 11: 558-572, 2011.

28. Tan N, Dai L, Liu X, Pan G, Chen H, Huang J and Xu Q: Upregulation of caprin1 expression is associated with poor prognosis in hepatocellular carcinoma. Pathol Res Pract 213: 1563-1567, 2017.

This work is licensed under a Creative Commons Attribution-NonCommercial-NoDerivatives 4.0 International (CC BY-NC-ND 4.0) License. 